serie

WP-AD 2011-07
Social vs. risk preferences under the veil of ignorance

Nicola Frignani and Giovanni Ponti 
Los documentos de trabajo del Ivie ofrecen un avance de los resultados de las investigaciones económicas en curso, con objeto de generar un proceso de discusión previo a su remisión a las revistas científicas. Al publicar este documento de trabajo, el Ivie no asume responsabilidad sobre su contenido.

Ivie working papers offer in advance the results of economic research under way in order to encourage a discussion process before sending them to scientific journals for their final publication. Ivie's decision to publish this working paper does not imply any responsibility for its content.

La Serie AD es continuadora de la labor iniciada por el Departamento de Fundamentos de Análisis Económico de la Universidad de Alicante en su colección "A DISCUSIÓN" y difunde trabajos de marcado contenido teórico. Esta serie es coordinada por Carmen Herrero.

The AD series, coordinated by Carmen Herrero, is a continuation of the work initiated by the Department of Economic Analysis of the Universidad de Alicante in its collection "A DISCUSIÓN", providing and distributing papers marked by their theoretical content.

Todos los documentos de trabajo están disponibles de forma gratuita en la web del Ivie http:/ / www.ivie.es, así como las instrucciones para los autores que desean publicar en nuestras series.

Working papers can be downloaded free of charge from the Ivie website http://www.ivie.es, as well as the instructions for authors who are interested in publishing in our series.

Edita / Published by: Instituto Valenciano de Investigaciones Económicas, S.A.

Depósito Legal / Legal Deposit no.: V-1491-2011

Impreso en España (marzo 2011) / Printed in Spain (March 2011) 


\title{
Social vs. risk preferences under the veil of ignorance*
}

\author{
Nicola Frignani and Giovanni Ponti*
}

\begin{abstract}
This paper reports experimental evidence from a Dictator Game experiment in which subjects choose repeatedly one out of four options involving a pair of fixed monetary prizes, one for them, one for another anonymously matched subject. In some sessions, player position (i.e. the identity of the best paid agent, constant across all options) is known in advance before subjects have to make their decision; in other sessions subjects choose "under the veil of ignorance", not knowing to which player position they will be eventually assigned. We also collect evidence from additional sessions in which the same options correspond to binary lotteries, in which subjects may win the high or the low prize, but their decisions do not affect other participants. We frame subjects' decisions within the realm of a simple mean-variance utility maximization problem, where the parameter associated to the variance is interpreted, depending on the treatment conditions, as a measure of pure risk aversion, pure inequality aversion, or some combination of the two. We also condition our estimates to subjects' individual socio-demographic characteristics.
\end{abstract}

Keywords: dictator games, social preferences, risk preferences, functional identification.

JEL Classification: D86.

\footnotetext{
"We are grateful to A. Cabrales, D. Di Cagno, G. Harrison, J. Kovarik, S. Mancinelli, E. Martínez, R. Miniaci, M. Piovesan, N. Piva, G. Rimoldi and L. Ruström for stimulating coments and suggestions. The usual disclaimers apply. Financial support from the Spanish Ministry of Education and Science (SEJ 2007-62656 and Consolider-Ingenio 2010, CSD2006-00016), MIUR (PRIN 2007MCKEYA), Generalitat Valenciana (Research Projects Grupos 03/086) and the Instituto Valenciano de Investigaciones Económicas (Ivie) is gratefully acknowledged.

${ }^{* *}$ N. Frignani: Università di Ferrara. G. Ponti: University of Alicante and Università di Ferrara. Corresponding author: giuba@merlin.fae.ua.es.
} 


\section{Introduction}

At first sight, risk and inequality aversion appear to be unrelated concepts. With the former, we refer to "the reluctance of a person to accept a bargain with an uncertain payoff rather than another bargain with a more certain, but possibly lower, expected payoff"; with the latter, "the preference for fairness and resistance to inequitable outcomes." ${ }^{1}$

However, when taking a closer look, these two concepts can be related on several grounds. The first, intuitive connection comes from the fact that they both rely on some measure of distance (or distribution) of outcomes. In this respect, a risk-averse individual is willing to accept a lower expected reward in search of a smaller outcome variability and -by the same tokenan inequity averse individual is willing to opt for a poorer society, if this implies less income inequality. The hypothetical frame of the veil of ignorance (VOI hereafter) is built exactly upon this intuitive similarity. Rousseau's [25] original position is, probably the first example of the VOI: the idea is to look at constitutional rules from the point of view of an outside observer, not knowing which will eventually be her player position (call it income, social status, opportunities) in the society. A similar viewpoint is taken by John Rawls' [24] influential book, The Theory of Justice, in which the VOI is applied to put forward the maxmin principle as the rational principle to implement social justice. As it is well known, this view has been strongly criticized by John Harsanyi's [17] treatment of the VOI, by which the utilitaristic approach is justified by the fact that the preferences of an "impartial and sympathetic" observer should be concerned with ". . . the welfare of each participant but having no partial bias in favor of any participant... (p. 49)". At the core of Harsanyi's argument lies the idea that it is possible to make distributional judgements taking expectations over purely self-interested preferences, once the probability (and the associated risk) of being any "participant" in the society is properly taken into account. In this respect, it is exactly the VOI hypothetical frame which provides purely self-interested preferences with a distributional taste.

Thirty years later, many economists are now used to think that distributional (or "social") preferences may well exist even without the fictitious frame of the VOI. This view has been largely influenced by the vast experimental literature showing that subjects, in many classic experimental protocols, exhibit social preferences, with a strong taste against inequality. ${ }^{2}$

\footnotetext{
${ }^{1}$ Both definitions are borrowed from the correponding entries of Wikipedia (http://www.wikipedia.org).

${ }^{2}$ This literature is well summarized in the surveys of Fehr and Schmidt [13] and Sobel
} 
This, implicitly, challenges Harsanyi's claim, unless it is provided a suitable frame in which we can disentangle risk from distributional concerns, when it comes to situations in which individuals have to choose among risky prospects which affect the well-being of others.

Whether individuals exhibit social preference when choosing under the VOI remains, essentially, an empirical matter. In this respect, there is a growing empirical literature which use questionnaire data to estimate risk and distributional preferences of the same subject pool. Paradigmatic is the case of Carlsson et al. [9], who measure subjects' preferences for risk and inequality through choices between imagined societies and lotteries. They also collect personal information on the socio-demographic characteristics of their subject pool, concluding that "... (even under the veil of ignorance) many people appear to have preferences regarding equality per se. We have also found that both relative risk aversion and inequality aversion vary with sex and political preferences. On average, women and left-wing voters have higher parameter values for both relative risk aversion and inequality aversion" (p. 391). ${ }^{3}$

In this literature, subjects mainly face hypothetical situations and, in this sense, their viewpoint resembles that of Harsanyi's "impartial and sympathetic observer". In this paper, we shall measure subjects' risk and distributional concerns relying on experimental methods. In particular, we estimate risk and inequality aversion using observations from subjects' actual decisions (that is, decisions which directly affect their own, together with others', financial rewards in the experiment). ${ }^{4}$ To this aim, we borrow the design (and part of the experimental evidence) from the work of Cabrales et al. [7] (CABRA hereafter), who set up a complex 3-phase experimental design to estimate subjects' social preferences (within the realm of Fehr and Schmidt's [12] model, F\&S hereafter) to explain (and predict) their behavior in a stylized (matching) labor market. Distributional preference parameters are estimated in the first phase of the experiment, a simple Dictator Game in which subjects repeatedly select their favorite option among a fixed menu of four, which changes at every round. Each option consists in two monetary prizes, one for them, one for another (randomly and anonymously matched) subject participating to the experiment.

In CABRA player position (i.e. the identity of the best paid agent) is constant across options, and known in advance before subjects have to make 
their decisions. In this paper, we complement their evidence $\left(T_{1}\right)$ with two additional treatments in which

1. subjects face the same sequence of decisions under the VOI, knowing ex-ante that either player position is equally likely $\left(T_{2}\right)$, or

2. the same sequence of decisions is made under a "lottery frame", in which player position is unknown (and equally likely), but there is no payoff externality on others $\left(T_{3}\right)$.

While CABRA employ F\&S to estimate subjects' purely distributional preferences, in this paper we posit a simple mean-variance utility model, where, depending on the treatments, the parameter associated with the variance measures pure inequality aversion $\left(T_{1}\right)$, pure risk aversion $\left(T_{3}\right)$ or, in the VOI treatment $T_{2}$, some combination of the two. In this respect, the exercise here is rather different from that of the literature cited above: we exploit the experimental methodology by designing specific economic environments in which, sometimes risk and inequality concerns are isolated, sometime they are combined, checking how these alternative decision frames affect the estimates of the same parameter, under the same statistical model. Finally, we also conditions our estimates to some socio-demographic characteristics we infer from a detailed questionnaire, administered to all subjects at the end of each section.

In summarizing our finding, we shall treat $T_{2}$ as our "control condition", where both risk and inequality concerns should supposedly guide subjects' decisions, checking whether in $T_{1}$ and $T_{3}$-when only one dimension is present at a time- the estimated parameters move significantly. In this respect, we find that both pooled and individual estimated parameter distributions in $T_{2}$ and $T_{3}$ are remarkably similar. By stark contrast, the estimated inequality aversion in $T_{1}$ is significantly lower. This would imply that we can simply relying on risk aversion to explain subjects' behavior under the VOI. Our individual regressions also show a significant variability in the estimated parameters, across subjects and treatments. When we use our questionnaire to give an account of this heterogeneity, we find that Social Capital variables have a greater impact on the estimated parameters than more traditional socio-demographics, such as disposable income or family wealth.

The remainder of this paper is arranged as follows. In Section 2 we briefly describe our experimental design, where in Section 3 we present the results of our econometric exercise. Finally, Section 4 concludes, followed by an Appendix containing the experimental instructions. 


\section{Experimental design}

In what follows, we describe the features of our experimental environment.

\subsection{Sessions}

We run 8 sessions under 3 different treatments: 3 sessions for each of the "Dictator Game" treatments, $T_{1}$ and $T_{2}, 2$ sessions for the "Lottery" treatment, $T_{3}$. All experimental sessions were conducted at the Laboratory of Theoretical and Experimental Economics (LaTEx), of the Universidad de Alicante. A total of 192 students (24 per session) were recruited among the undergraduate population of the Universidad de Alicante. The experimental sessions were computerized. ${ }^{5}$ Instructions were read aloud and we let subjects ask about any doubt they may have had. In all sessions (but those of the lottery treatment $T_{3}$ ), subjects were divided into two matching groups of 12 , with subjects from different matching groups never interacting with each other throughout the session. ${ }^{6}$

All treatments share the same basic layout. At the beginning of each round $t=1, \ldots, 24$, subjects are informed about the choice set $C_{t}=\left\{b^{k}: k=1, \ldots, 4\right\}$, constant across treatments. Each option $b^{k}=\left(b_{1}^{k}, b_{2}^{k}\right)$ assigns a fixed monetary prize $b_{i}^{k}$ to player $i=1,2$, with $b_{1}^{k} \geq b_{2}^{k}$, for all $k$. After subjects have selected their favorite options, all payoff relevant information is revealed and payoffs are distributed.

\subsection{Treatments}

We now explain the details of our 3 experimental conditions.

\subsection{1 $T_{1}:$ pure inequality (CABRA)}

In $T_{1}$, subjects choose their preferred option after being informed about the outcome of the iid draw which fixes the player (i.e. their relative) position for that pair and round. Remember that, since $b_{1}^{k} \geq b_{2}^{k}$, player $1(2)$ looks at the distributional problem implicit in the option choice from the viewpoint of

\footnotetext{
${ }^{5}$ The experiment was programmed and conducted with the software z-Tree (Fischbacher [14]). The complete set of instructions, translated into English, can be found in the Appendix.

${ }^{6}$ Given this design feature, we shall read the data under the assumption that the history of each matching group corresponds to an independent observation. Clearly, the same does not apply in case of $T_{3}$, in that each subject's experimental history corresponds to an independent observation.
} 
the (dis)advantaged player. Once choices are made, we employ a "Random Dictator" protocol (Harrison and McDaniel [16]) to determine the payoff relevant decision, in that another iid draw fixes the identity of the subject whose choice determines the monetary rewards for that pair and round. ${ }^{7}$

\subsection{2 $T_{2}:$ VOI}

In treatment $T_{2}$ we modify the control treatment $T_{1}$ by introducing the VOI. In this case, subjects only know that the ex-ante probability of being assigned to either player position equals $\frac{1}{2}$. Everything else is just as in $T_{1}$, in particular the fact that subjects alternate player and Dictator positions in a (iid) random fashion.

\subsection{3 $T_{3}:$ pure risk (lottery)}

Our lottery treatment $T_{3}$ replicates treatment $T_{2}$ without any payoff externality on others. In this case, player position is uncertain (and equally likely), but each subject decides in isolation.

\subsection{Financial rewards}

All monetary payoffs in the experiment were expressed in Spanish Pesetas (1 euro is approx. 166 ptas.). ${ }^{8}$ Subjects received 1.000 ptas. just to show up, to which they summed up all their cumulative earnings throughout the 24 rounds of the experiment. Average earnings were about 12 euros, for an experimental session lasting for approximately 45 minutes.

\subsection{The Questionnaire}

At the end of each session, subjects were asked to answer a detailed questionnaire from which we distilled the following variables, which are used in

\footnotetext{
${ }^{7}$ CABRA also consider a treatment in which $i$ ) only the Dictator chooses and ii) subjects are assigned to the same player positon at all time. They show that parameter distributions are basically identical to those of $T_{1}$, where the same conclusions also holds under our statistical frame (reslts are not reported here, but are available upon request).

${ }^{8}$ It is standard practice, for all experiments run in Alicante, to use Spanish ptas. as experimental currency. The reason for this design choice is twofold. First, it mitigates integer problems, compared with other currencies (USD or Euros, for example). On the other hand, although Spanish pesetas are no longer in use (substituted by the Euro in the year 2002), Spanish people still use Pesetas to express monetary values in their everyday life. In this respect, by using a "real" (as a opposed to an artificial) currency, we avoid the problem of framing the incentive structure of the experiment using a scale (e.g. "Experimental Currency") with no cognitive content.
} 
Section 3.3.

1. Gender $=1$ for females;

2. RiskLover $\in[0,1]$, indicating the relative frequency of "risky" choices in a series of 5 binary lotteries Holt and Laury's [18] style.

3. WeeklyBudget $\in[0,400]$. In Euro, a proxy of subjects' disposable income.

4. RoomSizeRatio: the ratio between number of rooms of the main residence and the number of the family members (i.e., a proxy of the family's wealth).

5. Satisfaction. "How do you feel in this moment with your life?". 7scaled answer from 0 ("Not at all satisfied") to 1 ("Very satisfied").

6. Felicity. "Taking everything into consideration, would you call yourself i) very happy $(\mathbf{F e l}=1)$; ii) quite happy $(\mathbf{F e l}=.5)$; iii) not very happy $(\mathbf{F e l}=0)$.

7. Inequality $\in\{0,1\}$. A classic test of concerns for inequality, contained in many Social Capital questionnaires. Q. "Consider the following situation: Two secretaries with the same age do exactly the same work. However, one of them earns 20 euros per week more than the other. The one that is paid more is more efficient and faster, while working. Do you believe it is fair that one earns more than the other?". IA=1 if the answer is no, and 0 otherwise.

8. Parents $\in\{0,1\}$. Another classic question. Choose between "Independently of the qualities and deficiencies of parents, they should always be loved and respected" $(\mathbf{P A R}=0)$ and "Parents who have not earned the love by their attitudes and behavior should not be loved" $(\mathbf{P A R}=1)$.

\section{Estimating social and risk preferences}

In what follows, $i$ (and $j$ ) identify our subjects in $T_{3}$ (matched in pair in $T_{1}$ and $\left.T_{2}\right)$. Let $\mu(k)=\frac{b_{1}^{k}+b_{2}^{k}}{2}$ and $\sigma(k)=\sqrt{\frac{\left(b_{1}^{k}-\mu(k)\right)^{2}+\left(b_{2}^{k}-\mu(k)\right)^{2}}{2}}$ denote the arithmetic mean and standard deviation of the monetary payoffs associated with option $k$, respectively. We assume that subject $i$ 's preferences by choosing option $k=1, \ldots 4$, are defined by the following 


\section{Definition 1 (Mean-Variance Preferences)}

$$
u_{i}(k)=v_{i}(k)-\gamma_{i} \sigma(k)+\varepsilon_{i}^{k}
$$

where $v_{i}(k)=b_{i}^{k}$ in treatment $T_{1}, v_{i}(k)=\mu(k)$ in treatments $T_{2}$ and $T_{3}$, and $\varepsilon_{i}^{k}$ is an idiosyncratic error term (with zero mean and fixed variance) to facilitate estimation. Definition 1 postulates that subjects evaluate options by way of a simple mean-variance utility function (with noise), where the interpretation of $\gamma_{i}$ depends on the treatment.

1. In treatment $T_{1}, \gamma_{i}$ measures a pure distributional concern. Since subjects are informed about their player position before they have to decide their favorite option, using F\&S' terminology, we can think of $\gamma_{i}$ as a direct measure "inequality aversion".

2. In $T_{3}$ subjects face ordinary binary lotteries. Therefore, in this case, $\gamma_{i}$ measures pure risk aversion (with $\gamma_{i}=0$ indicating the null hypothesis of risk-neutrality).

3. Also in $T_{2} i$ chooses among lotteries, but this decision has distributional consequences for player $j$, too. In this sense, we expect $\gamma_{i}$ to capture some combination of both effects, one related to risk, the other to inequality.

According to this notation, subject $i$ chooses contract $\hat{k}$ at round $t$ if

$$
\hat{k}=\arg \max _{k}\left\{u_{i}(k)\right\} .
$$

Under the assumption that the stochastic term $\varepsilon_{i}^{k}$ is iid with an extreme value distribution, the probability that individual $i$ chooses the contract $k$ at round $t$ is therefore

$$
\operatorname{Pr}\left(y_{i t}=\hat{k}\right)=\frac{\exp \left(u_{i}(\hat{k})\right)}{\sum_{k=1}^{4} \exp \left(u_{i}(k)\right)} .
$$

Notice that (2) allows for parameter heterogeneity across subjects. Thus, the iid assumption does not stem from neglected individual unobserved heterogeneity, and it is consistent with the random order of the four contracts in the choice set $C_{t}$. 


\subsection{Pool estimates}

Table 1 report estimates of $\gamma_{i}$ "pooled" by treatment, i.e. when (1) is estimated under the assumption that $\gamma_{i}$ is constant across treatment $T_{h}$ (i.e. $\gamma_{i}=g^{h}, h=1, \ldots, 3$ ). Since, in $T_{1}$, subjects choose their favorite option knowing their player position, we also provide pool estimates conditional on player position $i=1,2, g_{i}^{1}$. The reported estimated standard errors in Table 1 take into account matching group clustering.

\begin{tabular}{cccccc}
\hline \hline & Coeff. & Std. err. & $p$-value & \multicolumn{2}{c}{ 95\% conf. int. } \\
\hline$g^{1}$ & .267 & .304 & 0 & .208 & .327 \\
\hline$g_{1}^{1}$ & .871 & .394 & .027 & .098 & 1.644 \\
\hline$g_{2}^{1}$ & .208 & .039 & 0 & .132 & .285 \\
\hline$g^{2}$ & .347 & .0131 & 0.020 & .321 & .372 \\
\hline$g^{3}$ & .316 & .016 & 0 & .286 & .347 \\
\hline
\end{tabular}

Table 1: Pool estimates of $\gamma_{i}$

As Table 1 shows, all estimated parameters are significantly greater than zero, indicating inequality/risk aversion of our "representative subjects" in the three treatments. As for the comparison across treatments, we shall treat $T_{2}$ as our "control condition", where both risk and inequality concerns are supposed to guide subjects' decision, checking in $T_{1}$ and $T_{3}$, (if and) how the estimated parameter changes when only one dimension is present at a time. In this respect, Table 1 shows that $T_{2}$ is characterized by the highest value for $\gamma$, and $T_{1}$ by the highest dispersion. The only significant difference is that between between $g^{1}$ and $g^{2}(z=-2.4, p=.017)$, indicating that, in absence of any uncertainty about player position assignment, the estimated inequality aversion lowers significantly. By contrast, we cannot reject the null of equality between $g^{2}$ and $g^{3}$, suggesting that we could simply relying on risk aversion to explain subjects' behavior in $T_{2}$. Once, in $T_{1}$, we condition the estimate of $\gamma$ on player position, we find, consistently with Cabrales et al. [7], that "guilt" (i.e. sensitiveness to payoff difference when enjoying an advantageous relative position) is higher than "envy" (i.e. sensitiveness to player position when suffering the lower end of the stick), although the difference in not significant $(z=-1.61, p=.106)$.

\subsection{Individual Estimates}

Figure 1 reports the distributions of individual $\gamma_{i}$, disaggregated for treatment conditions, $T_{1}$ to $T_{3}$, using 24 observations for each individual estima- 
tion.

Figure 1 confirms our preliminary conclusions from Table 1 , where $T_{2}$ and $T_{3}$ distributions are remarkably similar (both are positively skewed, with very few subjects characterized by a negative estimated parameter). By contrast, the parameter distribution in $T_{3}$ display higher dispersion, and we also find a higher frequency of inequality "lovers" (i.e. subjects with $\gamma_{i}<0$ ) than those of $T_{2}$ and $T_{3}$. This should explain why the pooled estimate in $T_{1}$ is significantly lower.

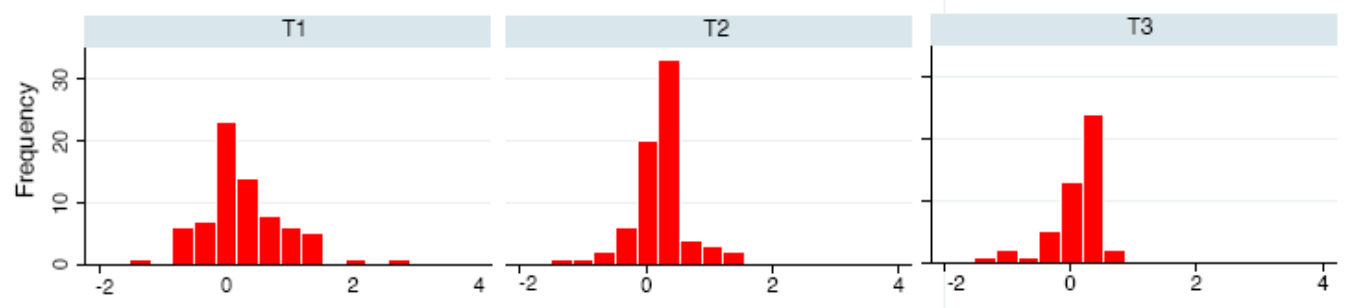

Figure 1. Individual estimates of $\gamma_{i}$

Clearly, Figure 1 cannot take into account the standard errors of the individual estimates. This is why, in Table 2, we summarize our results on subjects' preference heterogeneity by partitioning our subject pool, assigning each subject to a category (from "lover" to "averse") depending on whether or not her estimated $\gamma_{i}$ complies (at the 10\% confidence level) with the null hypothesis of neutrality to risk/inequality.

\begin{tabular}{|l|c|c|c|c|}
\hline & Lover & Neutral & Averse & Total \\
\hline \multirow{2}{*}{$T_{1}$} & $\mathbf{9}$ & $\mathbf{3 2}$ & $\mathbf{3 1}$ & $\mathbf{7 2}$ \\
& $12.5 \%$ & $44.44 \%$ & $43.06 \%$ & $100 \%$ \\
\hline \multirow{2}{*}{$T_{2}$} & $\mathbf{4}$ & $\mathbf{2 9}$ & $\mathbf{3 9}$ & $\mathbf{7 2}$ \\
& $5.56 \%$ & $40.28 \%$ & $54.17 \%$ & $100 \%$ \\
\hline \multirow{2}{*}{$T_{3}$} & $\mathbf{4}$ & $\mathbf{1 9}$ & $\mathbf{2 5}$ & $\mathbf{4 8}$ \\
& $8.33 \%$ & $39.58 \%$ & $52.08 \%$ & $100 \%$ \\
\hline \multirow{2}{*}{ Total } & $\mathbf{1 7}$ & $\mathbf{8 0}$ & $\mathbf{9 5}$ & $\mathbf{1 9 2}$ \\
& $8.85 \%$ & $41.67 \%$ & $49.48 \%$ & $100 \%$ \\
\hline
\end{tabular}

Table 2: Partitioning our subject pool with respect to their attitude toward inequality/risk 
Once again, Table 2 confirms previous analysis: we find an homogeneous partition in $T_{2}$ and $T_{3}$, while the relative frequency of inequality averse subjects in $T_{1}$ is about $10 \%$ lower.

\subsection{Socio-demographics and Social Capital}

As we already discussed in Section 2.4, we collected information on individual socio-demographics, risk and social attitudes. In Table 3, we condition the estimates of $\gamma_{i}$ upon this personal information. Again, also in Table 3 standard errors are adjusted to take into account matching group clustering.

\begin{tabular}{|l|c|c|c|c|c|c|c|}
\hline & Gen & RL & Sat & Fel & Par & Ineq & const. \\
\hline \multirow{2}{*}{$T_{1}$} & .104 & .077 & .339 & -.192 & .018 & .44 & .09 \\
& $(.375)$ & $(.394)$ & $(.09)^{* * *}$ & $(.371)$ & $(.384)$ & $(.529)$ & $(.325)$ \\
\hline \multirow{2}{*}{$T_{2}$} & -.05 & -.148 & -.05 & .04 & .016 & .06 & .356 \\
& $(.015)^{* * *}$ & $(.07)^{* *}$ & $(.046)$ & $(.07)$ & $(.019)$ & $(.03)^{* *}$ & $(.07)^{* * *}$ \\
\hline \multirow{2}{*}{$T_{3}$} & .033 & -.262 & .058 & -.21 & .071 & .013 & .433 \\
& $(.041)$ & $(.11)^{* *}$ & $(.05)$ & $(.09)^{* *}$ & $(.04)^{*}$ & $(.05)$ & $(.1)^{* * *}$ \\
\hline
\end{tabular}

Table 3: Estimates of $\gamma_{i}$ conditional on socio-demographics. Standard errors between brackets. ${ }^{* * *}=1 \%$ significant; ${ }^{* *}=5 \%$ significant; $*=10 \%$ significant.

In what follows, we shall comment on the role of each explanatory variable in the three regressions.

1. gender is (negative and) significant in $T_{2}$ only. Our negative coefficient contradicts the reported evidence in Carlsson et al. [9], in that women, under the VOI, exhibit lower risk/inequality aversion than men. ${ }^{9}$ As for the relation between gender and risk aversion, despite the (negative and) significant correlation between gender and RL (coeff. $-0.285, p=0$ ), women in $T_{3}$ do not show higher risk aversion.

2. $\mathbf{R L}$ is negative and significant for all treatments, except for $T_{1}$, the one in which subjects face no risk whatsoever. While the coefficient for $T_{3}$ is easy to rationalize (less risk averse subjects according to the questionnaire, also reveal lower risk aversion thought their experimental decisions), the (negative and) significant impact of RL in $T_{2}$ is consistent with our previous results, in that the estimate of $\gamma$ in $T_{2}$ appears to depend more on risk, rather than inequality, aversion.

\footnotetext{
${ }^{9}$ Croson and Gneezy's [11] survey on gender differences in preferences warns that women's altruistic motives are not uniformly stronger than those of men, but simply more situationally specific.
} 
3. Social Capital proxies. While other "classic" socio-demographics variables -such as subjects' disposable income, or wealth, proxied by WB and RSR- are never significant (and we omit to report the corresponding estimates in Table 3), other Social Capital proxies, mostly related to subjects' self assessed overall well-being state, affect our estimation in unexpected directions. For example, high Sat pushes up inequality aversion in $T_{1}$, while high Fel pushes down risk aversion in $T_{3}$. On the other hand, Par and Ineq are (positive and) significant only in case of $T_{3}$ and $T_{2}$, respectively.

4. Constant. Positive and (highly) significant in case of $T_{2}$ and $T_{3}$ only, showing the absence of a significant "treatment effect" in the measurement of $\gamma$ in $T_{1}$, once we controlled for the other variables reported in Table 3.

\section{Conclusion}

Our experimental evidence suggests that, under the VOI, subjects' aversion to outcome variability mostly reflects risk, rather than distributional, concerns, although both components, when operating alone, are significant motivators to action. We also see that subjects' heterogeneity is well captured by some Social Capital proxies, which more and more often complement standard socio-demographic variables, and that the same personal characteristics operate in rather different ways, depending on whether only risk or inequality characterizes the choice environment, or both. ${ }^{10}$ In addition, typical proxies for risk attitude (such as our RL variable) have a significant impact in subjects' distributional choices, so as typical Social Capital proxies have a significant impact on purely risky decisions. All this evidence seems to suggest that risk and distributional concerns are not independent behavioral phenomena, and that their intrinsic relation is more complex than what is usually assumed. ${ }^{11}$

In a recent paper, Andersen et al. [2] find that joint elicitation of risk and time preferences provides estimates of discount rates that are significantly lower than those put forward by previous literature. While they reach this conclusion by disentangling risk and time preferences by functional identification, what we find here is somewhat similar, in that we show that

\footnotetext{
${ }^{10}$ In this respect, our findings are in line with those of Capra et al. [8].

${ }^{11}$ Along a similar vein, Kovarik [21] shows how altruistic motives decay when monetary consequences move further away in the future.
} 
distributional concerns may well vary, depending on the degree of uncertainty that characterizes the choice environment. It may be worthwhile to remember that most of the discussion on distributional preferences has been built on evidence of experimental protocols (such as the Dictator Game) in which subjects face no uncertainty whatsoever. By introducing uncertainty in the simplest possible way (via totally exogenous random devices), what we observe is a significant variation in our estimations.

These considerations may have important behavioral consequences, well beyond the -somehow artificial- frame of the VOI. Consider, like in Karni et al. [20], situations in which subjects unilaterally decide on the distribution of the probabilities with which an indivisible prize is to be allocated to them, rather than to (an)other subject(s). ${ }^{12}$ Or, like in Bolton et al. [5], situations in which random devices are used to implement distributional justice. Last, but not least, in more complex environments -such as Ultimatum Gamesin which distributional concerns interact with strategic uncertainty. ${ }^{13}$

We conclude by indicating an interesting avenue for future research, which is related to the distribution of risk across subjects (i.e., what is usually defined as aggregate risk). In our VOI treatment, risk is (perfectly and) negatively correlated across subjects, in the sense that being assigned to one player position directly implies the assignment of the other player position to the other couplemate. This is a situation in which aggregate risk is completely absent, in that any given option always distributes -within the pair- the same financial reward. Consider, instead, the case in which player assignments were completely independent, or (perfectly and) positively correlated. ${ }^{14}$ In the latter case, any model of distributional preferences a' $l a$ $\mathrm{F} \& \mathrm{~S}$ would predict no difference in behavior, when compared with ordinary lotteries, like our $T_{3}$. On the other hand, subjects may indeed show social preferences by controlling, through their decision, the aggregate risk sharing distribution within the group.

\footnotetext{
${ }^{12}$ Unlike Andreoni and Miller (2000) or Fisman et al. [15], whose protocols involves subjects allocating tokens between themselves and other subject(s) in a deterministic fashion, in Karni et al. [20] subjects allocate probabilities (i.e., once again, risk and distributional concerns are difficult to identify).

${ }^{13}$ Take, for example, Prasnikar and Roth [23] or Binmore et al. [4].

${ }^{14}$ These two latter environments are exactly those explored in Kroll and Davidovitz [22], in a very original experimental protocol, using as subjects pool primary shool kids.
} 


\section{References}

[1] Amiel Y, Creedy J and Hurn S (1999). "Measuring attitudes towards inequality", Scandinavian Journal of Economics, 101, 83-96.

[2] Andersen S, Harrison GW, Lau MI and Rutström E (2008). "Eliciting Risk and Time Preferences", Econometrica 76 , 583-618.

[3] Andreoni J and Miller J (2002). "Giving According to GARP: An Experimental Test of the Consistency of Preferences for Altruism", Econometrica, 70, 737-753.

[4] Binmore K, McCarthy J, Ponti G, Samuelson L and Shaked A (2002), "A Backward Induction Experiment", Journal of Economic Theory, $104,48-88$.

[5] Bolton G, Brandts J. and Ockenfels A. (2005). "Fair Procedures: Evidence from Games Involving Lotteries", Economic Journal, 115, 10541076 .

[6] Bosmans K and Schokkaert E (2003). Social Welfare, the Veil of Ignorance and Purely Individual Risk: An Empirical Examination, mimeo.

[7] Cabrales A, Miniaci R, Piovesan M and Ponti G (2008), Social Preferences and Strategic Uncertainty: an Experiment on Markets and Contracts, American Economic Review, forthcoming.

[8] Capra CM, Lanier K, Meer S (2008). Attitudinal and Behavioral Measures of trust: a NewComparison, Emory University, mimeo.

[9] Carlsson F , Daruvala F, Johansson-Stenman O (2005). "Are People Inequality-Averse, or Just Risk-Averse?", Economica, 72, 375-396.

[10] Cowell, F. and Schokkaert, E. (2001), "Risk perceptions and distributional judgements", European Economic Review, 45, 941-952.

[11] Croson R. and Gneezy U. (2008). "Gender differences in Preferences", Journal of Economic Literature, 47, 1-27.

[12] Fehr E and Schmidt KM (1999). "A theory of fairness,competition and cooperation", Quarterly Journal of Economics, 114, 817 -868.

[13] Fehr E and Schmidt KM (2003). "Theories of Fairness and Reciprocity: Evidence and Economic Applications." in Dewatripont M, Hansen LP 
and Turnovsky SJ ( eds.), Advances in Economics and Econometrics: Theory and Applications, Eighth World Congress, Vol I: 208-257. Cambridge UK, Cambridge University Press.

[14] Fischbacher U (2007). "z-Tree: Zurich Toolbox for Ready-made Economic Experiments." Experimental Economics, 10(2): 171-178.

[15] Fisman R, Kariv S and Markovits D (2007). "Individual Preferences for Giving". American Economic Review 97(5): 1858-76.

[16] Harrison GW and McDaniel T (2008). "Voting Games and Computational Complexity", Oxford Economic Papers, 60, 546-565.

[17] Harsanyi, J. C. (1977), Rational Behavior and Bargaining Equilibrium in Games and Social Situations, Cambridge University Press, Cambridge.

[18] Holt CA and Laury SK (2002). "Risk Aversion and Incentive Effects", American Economic Review, 92(5), 1644-55.

[19] Hörisch A. (2007). Is the veil of ignorance only a concept about risk? An experiment, University of Munich Discussion paper 2007-04.

[20] Karni E, Salmon T and Sopher B (2008). "Individual sense of fairness: an experimental study", Experimental Economics, forthcoming.

[21] Kovarik J (2009), "Giving it now or later: Altruism and discounting", Economic Letters, forthcoming.

[22] Kroll Y and Davidovitz L (1999). Choices in Egalitarian Distribution: Inequality Aversion versus Risk Aversion, LSE CERD Discussion Paper n. $43 / 1999$.

[23] Prasnikar V and Roth A (1992). "Considerations of fairness and strategy: Experimental data from sequential games", Quarterly Journal of Economics, 107, 865-888.

[24] Rawls J (1971). A Theory of Justice, Cambridge, Mass., Harvard University Press.

[25] Rousseau JJ (1762). The Social Contract, London, Dent, 1973.

[26] Sobel J (2005). "Interdependent Preferences and Reciprocity," Journal of Economic Literature, 43, 392-436. 


\section{PUBLISHED ISSUES *}

WP-AD 2011-01 "Solving the multi-country real business cycle model using ergodic set methods" S. Maliar, L. Maliar, K. Judd. January 2011.

WP-AD 2011-02 “Anti-piracy policy and quality differential in markets for information goods” J.M. López-Cuñat, F. Martínez-Sánchez. January 2011.

WP-AD 2011-03 "Loyalty discounts"

U. Akgun, I. Chioveanu. February 2011.

WP-AD 2011-04 "Recreation, home production, and intertemporal substitution of female labor supply: evidence on the intensive margin"

J. González Chapela. February 2011.

WP-AD 2011-05 “On the effects of deposit insurance and observability on bank runs: an experimental study"

H.J. Kiss, I. Rodríguez-Lara, A. Rosa-García. February 2011.

WP-AD 2011-06 “The role of public and private information in a laboratory financial market” S. Alfarano, E. Camacho, A. Morone. February 2011.

WP-AD 2011-07 "Social vs. risk preferences under the veil of ignorance" N. Frignani, G. Ponti. March 2011.

WP-AD 2011-08 “A model of music piracy with popularity-dependent copying costs” A. Piolatto, F. Schuett. March 2011.

WP-AD 2011-09 "Discrimination in second hand consumer markets: evidence from a field experiment” M. Bosch, M.B. Cobacho. March 2011.

WP-AD 2011-10 "Rental housing discrimination and the persistence of ethnic enclaves" M. Bosch, M.A. Carnero, L. Farré. March 2011.

WP-AD 2011-11 “Labour status and involuntary employment: family ties and part-time work in Spain” A. Denia, M.D. Guilló. March 2011.

\footnotetext{
* Please contact Ivie's Publications Department to obtain a list of publications previous to 2011.
} 


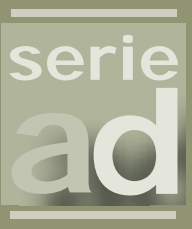

\section{I vie}

Guardia Civil, 22 - Esc. 2, 1 o

46020 Valencia - Spain

Phone: +34963190050

Fax: +34 963190055

Department of Economics

University of Alicante

Campus San Vicente del Raspeig

03071 Alicante - Spain

Phone: +34965 903563

Fax: +34965903898

Website: www.ivie.es

E-mail: publicaciones@ivie.es 